\title{
Eleven Ways to Critique an Article
}

\section{Mike Metcalfe}

University Of South Australia

Adelaide, South Australia, 5000

mike.metcalfe@unisa.edu.au

Good critiquing requires insight, asking thoughtful questions and seeing behind the text. How can someone practice this art? This paper aims to provide some pointers. Having been involved with critiquing articles with systems-thinking Ph.D. candidates for a number of years, I have attempted to expand on the ancient, yet wonderful questions, "What is their argument'?" and "Is their conclusion justified?", as a critiquing method using perspectives such as systems-thinking, evolution theory, picturing, dialectic and the perspectival thinking.

My approach to critiquing articles comes from trying to develop ways to appreciate, and research complex social problems, such as the design of a socio-technical system. Learning how to critique past actions is part of that. The approach taken in this paper aligns with $\mathrm{C}$. West Churchman's advice that new knowledge can also come from finding new perspectives, which can give you more choices. I hope to suggest a few new perspectives on critiquing an article, and so provide a few new choices. But more importantly the intent is to encourage a habit of perspectival thinking.

This paper will be presented in two parts, each one describing different perspectives that can direct a critique. The eleven perspectives presented will begin with a very brief summary of the supporting literature, followed by a set of sample questions that may help you to adopt a particular perspective in your thinking about whatever article you wish to critique.

Of course, critiquing others' work is only the first step to writing your own articles. In Part 2 this 'flipping of the coin' from critique to writing will be discussed. I am not aware of many other authors who have provided an approach similar to that presented here. The closest 1 am aware of can be found at the web site of The Writing Centre at Harvard University. ${ }^{2}$ They, understandably, focus on extracting a justified argument, rather than including perspectival thinking.

Why do I use the word 'critique?' I am not assuming the word 'critique' has an immoveable definition. I am using it in the sense of 'constructive evaluation' or 'literary review', hoping to include concepts like, 'lessons learnt' and 'how it might be done differently next time'. I have chosen it over words like 'review', 'analyse', 'evaluate' and 'discuss', because I find them too vague or too mathematical. This paper is my explanation of 'critique' by raising questions from an explicit perspective.

In terms of process, I am assuming that undertaking a critique involves reading an article, using the questions listed in this paper to think of an overall argument you wish to make, and writing it into a 'critique essay' which itself has a very explicit, welljustified, conclusion (the argument). 
Why undertake a critique? The intent of a critique is for learning to take place, yours and, where feedback is possible, the author of article. So, the way you go about critiquing an article needs to include methodically opening up the way you think. This means two 'learnings' need to take place. One is about the article being critiqued and the other is how best to undertake the critiquing process. This paper provides examples for beginners but you should develop your own critique perspective and resulting set of questions. Alternatively you may think about how the questions can be used. By improving the critique process, you will increase or get a better understanding of what you learn from the articles.

Why articles? The critique methods mentioned in this paper can be applied also to books, chapters, lectures, courses, projects, human inquiry, human activity problems, and policy. The term 'article' was used only for brevity.

\section{Part 1: Perspectives No. 1-6}

\section{The Simple-Argument Perspective}

\section{Background}

This style of critique draws on the 'argument as inquiry' perspective, which in modern times is attributed to Popper's [1963] Conjecture and Refutations, Perelman's [1989], The New Rhetoric, Walton's [1998] The New Dialectic, and van Eemeren's [1987] Handbook of Argumentation Theory. By 'argument' it is not meant formal logic nor quarrels but rather reasoned debate where an article is required to have an explicit upfront conclusion (the argument line) that needs to be justified with supporting evidence. The article should lay out this evidence in the form of reasoning and/or observations (descriptive or measurement). Counter arguments need to be anticipated and satisfactorily dealt with, otherwise the conclusion will not be convincing to the critiquer. Critiquing an article using this perspective involves evaluating the argument and supporting evidence.

Start by looking for the one sentence argument (conclusion, point) of an article-it should be presented in the article's abstract, the introduction and the conclusion. The conclusion is often the safest place to look. Care needs to be taken to compare the explicit and implicit argument. The authors may clearly state an argument but you may finish reading the article under the impression there was an alternative implicit argument. The argument also needs to have some surprise value, be a little insightful or, as Popper argues, be risky, falsifiable. Arguing the sky is blue would not be very insightful; arguing that it was red in the early stages of the earth's development might be. The innovation may be in the evidence. If you had novel evidence that the sky was blue, that may be convincing.

An article should not fail to convince you that its conclusion is justified merely because of poor definition of some key words. Technology, sociology and medicine are disciplines that have developed their own extensive vocabulary. History has not. It is 
the job of the author to communicate clearly with the intended audience, so an article can be criticised if it uses ill-defined terms. The greatest danger occurs when a word has several meanings and the reader is not alerted to that which the author is using. For example, the word 'critical' means negative, exact, nuclear, urgent and emancipatory. Scientists have spent many centuries defining their 'technical' terms but in social inquiry an author may need to spend some space defining, bounding and contrasting terms and concepts.

A reasoned argument needs at least two people. With article critique, this will be the author(s) presenting his or her argument to you. The arguers need to introduce themselves, their expertise in this area, their motivation for writing the paper, the motivation for why you might want to bother reading the article and to acknowledge they are presenting a justified conclusion. This background may assist in your acceptance of their evidence. It should also assist you in anticipating where their evidence may be weak.

The quality of evidence is not a simple or absolute thing. Science likes very exact measurement, social inquiry likes real insight and to treat the collection of observations as a learning-by-doing action that assist the brain to find insights. The only suggestion I have to help you decide whether or not the evidence is adequate is to simply ask if it would be convincing to a knowledgeable audience.

\section{Critique Questions (Argument Perspective)}

Ask yourself the following questions about the article.

\section{Argument}

- What is the explicit or implicit argument (conclusion) of the paper? Was it stated upfront?

- What was their insight, i.e., was the argument novel, risky, open to falsification?

\section{Definitions}

- Are all key words well-defined (described)?

\section{Arguers}

- Who are the authors?

- Have they established their expertise?

- Why have they selected this particular argument?

\section{Evidence}

- What evidence is brought to support the argument (conclusion)?

- Was this evidence convincing, novel, insightful?

- Was the counter argument fully considered?

- Were there any observations? If so, why? Should there be?

\section{Audience}

- Who is the intended audience?

- Is the paper explicitly persuasive to this audience? 
Motivation

- Is the importance of the argument fully explained?

- What was the problem?

- Is it an important problem?

- What did you learn from the article?

- How could you use it to improve people's lives?

\section{The Systems-for-Thinking Perspective}

There are numerous species to this now very diverse genus called 'systems-thinking'. The particular species I am interested in seems to be traceable in the U.S. to Boulding's General Systems Theory: the Skeleton of Science [1961], Churchman's The Design of Inquiry Systems [1971] and his students Mason, Mitroff, Ackoff and Ulrich who have produced work similar to Weick and Argyris. On the other side of the Atlantic, Checkland [2000] strengthen the meme with his soft systems. The important point being that these writers see systems-thinking as a broad scope, fairly generic approach, to appreciating human activity problems. An article can be seen as a human activity problem and its critique as a problem-solving exercise. If the article is about research into some physical problem, like the overall mass of the universe, rather than a social one, like saving the environment, the systems-thinking critique method may be less appropriate.

While still a developing concept, my interpretation of systems-thinking for solving social problems is that it encourages these types of problems to be seen using five constructs. These include purpose, interconnectivity, connectivity, boundary, seeking new perspectives and learning from doing. These problem-solving constructs can be used to critique an article.

\section{Critique Questions (Systems Thinking Perspective)}

Ask yourself the following questions about the article.

Purpose

- What purpose has the author had in writing the article?

- How else might the author's purpose have been achieved?

- What purpose will the critiquer give to the paper?

- What purpose do you think those participating had?

- What purpose will they give to the article?

Inter-Connectivity

- What is the article connected to?

- What is its place in the literature, discipline or topic?

- What else has the author done?

- Is the conclusion unique? 
-What does the paper remind you of, how does it sit with what else you know?

- What other evidence is available?

- What ripple effects will it have on wider systems?

- What do you see as the wider system on which it will have the largest impact?

- What genre, inquiry tradition, and school of thought is it from?

Boundary

- Is the article complete, does it present a self-contained story?

- Are all the issues and concepts raised well-defined and scoped?

\section{Transformation (Action and Learning)}

- Does the date of the article matter?

- What is the pattern of events that lead to this article?

- What does this article change in my thinking (lessons learnt)?

- What were the inputs to the article?

- What skill level was required of the reader?

- What data (empirical and previous literature) was the article founded upon?

Does the article process these inputs well?

- Are the outputs of the article, the conclusion and recommendations fully justified?

Stakeholders' Perspectives

- Does the article give all stakeholders a voice, does it seek the perspective of all involved?

- Are the stakeholders' perspectives critiqued and/or justified?

\section{The Picturing Perspective}

This approach to critiquing an article involves drawing a picture of the problem represented by the article. You may want to explore different ways of visually representing articles. One approach which uses cartoon like chartography is Checkland's [2000] 'rich pictures'. Bronte Stewart [1999] provides some more examples. The usefulness of picturing is usually in the process of drawing the picture rather than the finished picture. The intent is to encourage appreciation of the problem in the article and to provide a way to reveal that appreciation to others.

First, establish what the problem is the article is addressing. Draw the people involved in the problem domain discussed in the article. Then add any 'things' such as organisations and equipment. Then draws arrows to depict relationships between what has been drawn so far. Indicate if these relationships are friendly or in conflict. 
A Simple Example

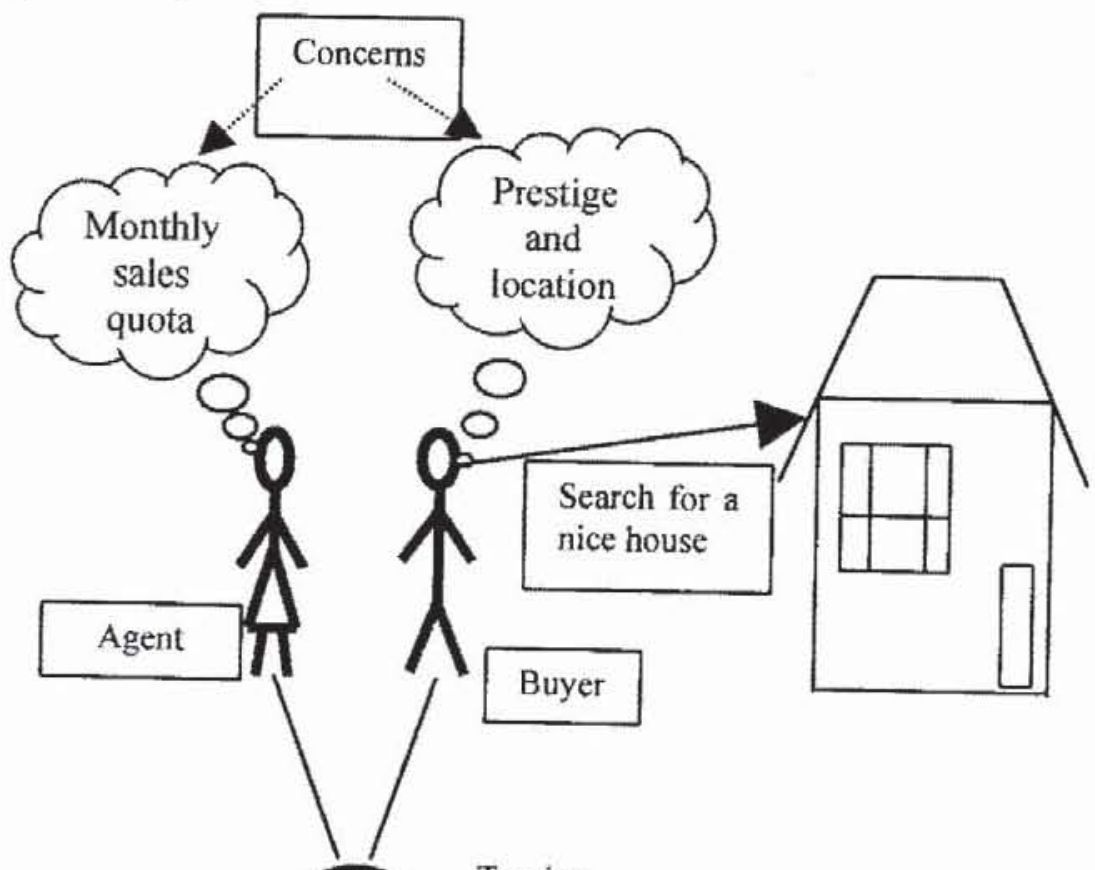

$\because$

Tension

Figure 1: A 'buying a house' rich picture.

A More Detailed Example

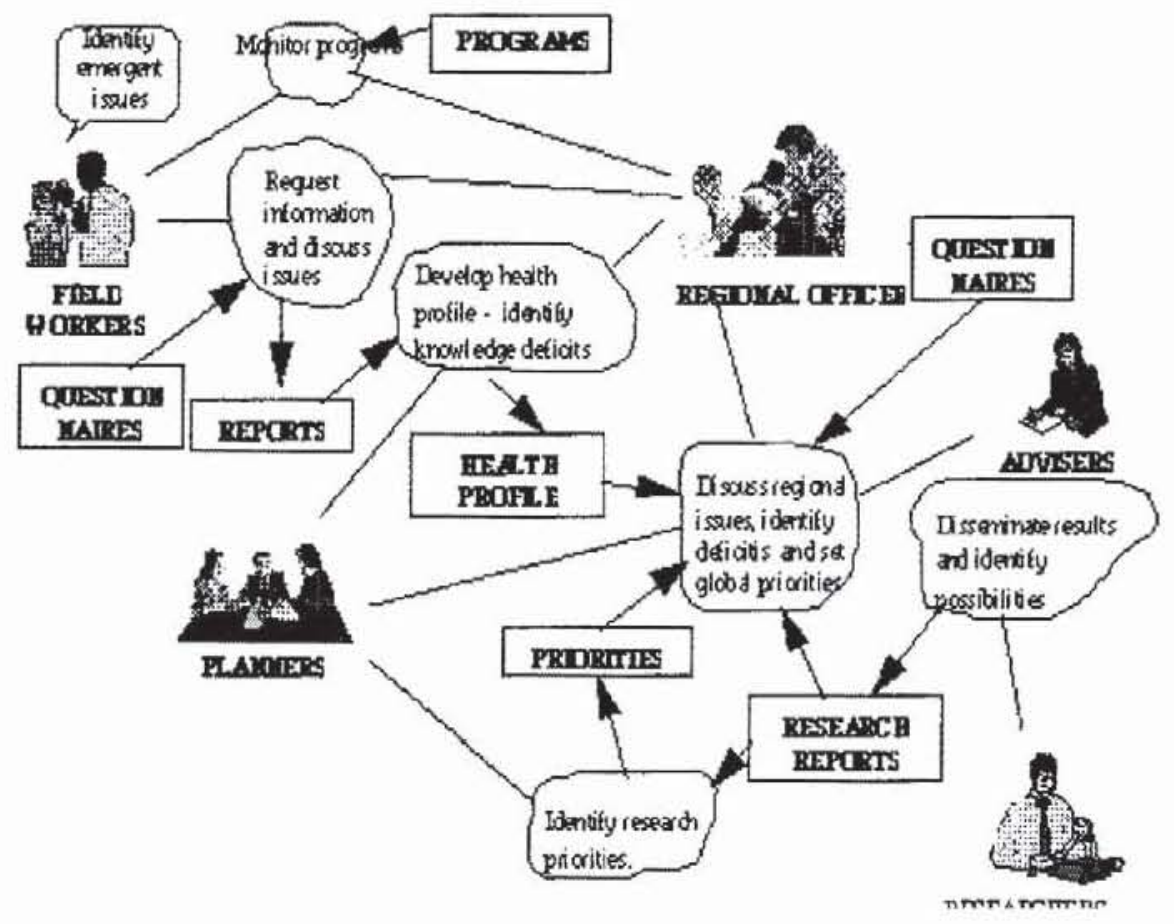




\section{The T.O.P. Perspective}

Linstone, [1984, 1993, 1999] sometimes with Mitroff [1993], has suggested that complex social situations need to be critiqued from three perspectives, a technical (T), organisational $(\mathrm{O})$ and personal $(\mathrm{P})$ perspective. For critiquing, this means that you can reflect on which perspective the article has been written from, and how it would be different if written from another perspective. For example, the space shuttle Challenger disaster can be presented as a mechanical $(\mathrm{T})$ failure, or as a failure of a few individuals $(\mathrm{P})$ making bad decisions, or as an organisational culture $(\mathrm{O})$ issue with managerialism overriding the engineering culture. An article may present one, two or all three of these perspectives.

\section{T.O.P Questions}

Ask yourself the following questions about the article.

- Is the article about people, organisations or things?

- How would it have been different if it were from a different perspective?

- Have the different perspectives of the people and/or cultures being provided?

- Have the people or cultural norms been treated as 'things?' or as intelligent and experienced people who can inform the author?

- Have all stakeholders had a voice?

- Has the author treated the problem addressed in the article as one that can usefully be solved by using scientific methods, i.e., taken a technical perspec tive? If so:

- Is the problem usefully subdivided into parts that can be measured?

- Is the situation repeatable so the measurements can be confirmed?

- Is it realistic to exclude any variables that have been excluded?

- Which stakeholder is to judge the solution to be valid?

- Is the evidence provided direct empirical or experiential?

- Has the author treated the problem addressed in the article as best being solved by appreciating the perspectives of the stakeholders $(\mathrm{P})$ ? If so:

- Do you get to hear their perspectives in their own words?

- How were opinions justified?

- Was the author cynical of whether the stakeholders understood their own minds or actions?

- Was any confirming evidence sought?

- What did stakeholders think of other stakeholders' perspectives?

- If a cultural norms perspective is being taken, is there a clear distinction between cultural and personality? For example,

- Does the author assume the culture is more than the personality of a few dominant leaders?

- Are the emergent properties of the culture identified and relevant? 
- How universal is the culture, are there contradictory sub cultures?

\section{The Concern's Perspectives}

Generalising from the TOP approach, the more generic perspective draws on the work of Kuhn's [1970] ideas of theory laden observation, Popper's expectations [1963], Churchman [1971] multiple inquiry methods, Linstone's [1999] multiple perspectives concepts and Haynes' [2000] Perspectival Thinking which draws on Polanyi, Hegel and Heidegger. The underlying theory of knowledge is that it is useful to identify two types of knowledge. The scientific type that produces 'objective facts' is well enough known. The alternative is that knowledge is perspectival. New knowledge means finding a new perspective. Kuhn uses the word 'paradigm' to describe this.

This dual knowledge approach believes that it is informative to separate inquiry into the 'thing' being inquired about from how it is being perceived. To take a simple example, I might study an organisation (the object) from a managerial efficiency perspective, a learning perspective or as a source of reliable income ( 3 possible perspectives). Critique involves attempting to separate these two types of knowledge, the thing being studied from the perspective.

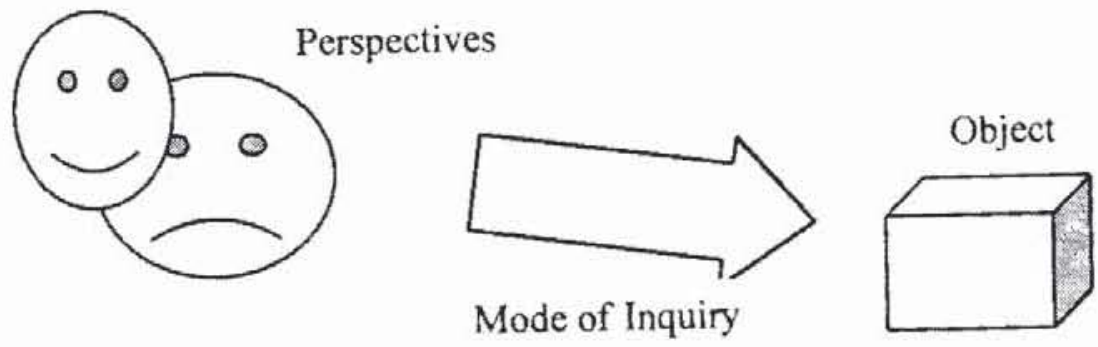

Two broad types of perspective have been identified. One is linguistic-from strong metaphors [Morgan 1986]. The other sort is more implicit and personal. Unprompted, people perceive new problem situations in different ways depending on their past experiences and their values. Terms like 'real interests', 'worries', 'theories of action' and 'people's concerns' align with this concept of an implicit perspective formation that make us pre-judge, or appreciate situations in certain ways. A critique might try to identify the concerns or primary perspective of the author (or maybe some participants in the article).

\section{Perspectival Questions}

Ask yourself the following questions about the article.

- What is the article about, what is the thing, the object under consideration? Think of this thing as being a system and ask yourself the system critique questions: 
- Are its boundaries well defined, what can it be contrasted with?

- What is its purpose?

- What does it change over time?

- What is it connected to?

-Who are the stakeholders?

- What perspective is being taken of the object under study?

- What are the origins of this perspective?

- Is it an external perspective or a concern?

- What are the limits of this perspective?

- Is it part of a wider perspective?

- What other perspectives might have been taken of the object under study?

\section{The Observation-as-Action Perspective}

There appears to be some very separate understandings about the role of observation (including sound, touch, smell and taste) in research. Some philosophers and critical theorists seem to think observations are more trouble than they are worth, rarely using them in research. Reasoning and thought experiments are considered sufficient to create useful knowledge. Measurement problems, the lack of reliability of the human senses and a lack of explanation of why the observation occurred contributes to this attitude. Moreover, Marx, who has influenced many social theorists, has a particular position on observation [Sowell, 1985, chp.2]. While Marx called his work empirical, he was not interested in the mere appearance of something. Rather first it is important to understand the underlying processes in tension going on behind the appearance of something. For example, a married couple can be living, eating, sleeping together but not be happy with their relationship, to understand the marriage you need to understand the underlying tensions. The same is true of a caterpillar; you cannot explain the appearance of a caterpillar unless you understand it is about to change into a butterfly. Put another way we have to learn to be able to see. A baby has to learn which sets of colors and shapes makes up a tree and a surgeon has to learn he or she is seeing when he/she opens up a body. Social researchers have to reason what is going on behind an observation. This can be used to critique observations by asking what underlying tensions has caused the thing you are looking at to be there as it is.

This lack of centrality of observation to research can also be found in some areas of science. Einstein's work was mainly mathematical and mind experiments (anologies). His 1939 book only draws on analogies as aid to reasoning. How the author perceives or uses observation provides a perspective with which to critique an article. You can ask whether the author explicitly uses reasoning or observational evidence to convince you of the conclusion.

However, in the positivist tradition, observations are central, especially the experiment. So you can ask if precision of measurement is considered insightful observation. Does the author display observation or measurement skills greater than a lay person or common sense? If so, then these are the hallmarks of the positivist scientist. Further, 
does the style of the author suggest that they are reporting the truth not a perspective, and that there is only one correct perspective? This is usually associated with the assumption that the observations can be repeated to get the same result and the observer is independent and unbiased.

The American Pragmatism approach to observation seems to be slightly different. It sees observations as problem solving by action, an experience, which enables the brain to draw on more analogies. The emphasis is not on seeking independent objective observations but to improve reasoning from action, including think of different ways to perceive the problem. This emphasis has been taken up in the action science, action research and learning from doing literature. In particular, Weick [1983] Argyris and Schon [1996], Ackoff [2000] and Checkland [2000] have taken it up as a problem appreciation method characterised by the learning or reflective loops approach. It advocates learning from a series of small trial and error learning loops rather than the 'one big plan' approach. Look for these assumptions in the observations in an article.

\section{Observational Questions}

Ask yourself the following questions about the article:

Why Observations?

- Why does the article have observations, why does it not?

- Are the observations intended to provide objective knowledge or merely to assist thinking?

- Could any observations be replaced with an analogy, reasoning or mind experiment?

- Do the observations convince you of the articles conclusion any more than the reasoning evidence did?

Underlying Tensions Observations

- What are the underlying tensions or forces that produced the 'thing' you are looking at as it now appears before you?

Observations for objective knowledge

- Are the authors seeking the one truth or do they acknowledge alternative interpretations of the observations?

- Do the observations produce convincing, objective knowledge?

- Are the observations repeatable?

- Are the observations generalisable to many other situations, will they remain valid across time and universally around the world?

- Are the observations all the evidence that is provided towards the conclusion?

- Is there any acknowledgement that observations are 'theory laden'?

Observations for learning from action

- Were the actions that produced the observations seen as an exercise in learning from action?

- Was there any attempt to undertake a series of small actions and reflect on each so as to redirect future actions to collect observations? 
- Is there any evidence of the author or others changing their perspective as a result of the observations?

- Have all stakeholders been given an opportunity to provide their perspective on the actions that created the observations?

- What other modes of inquiry (action) might have provided other learning?

- Was there any reflection as a result of the actions against the perspective (intellectual frame, theoretical construct) informing the observations?

\section{In Summation}

This paper has presented 6 ways to look at articles, six sets of criteria against which to think about the authors approach and underlying assumptions. In Part II another 5 perspectives will be presented, the last of which pulls together the previous 10 perspectives. The perspectives are not intended to be some kind of definitive set. They are all merely samples in need of improvement and modification. The important issue is that when you decide to question something, you ask yourself what underlying assumptions are driving your questions.

The perspectives in Part 2 are labelled

- The Metaphoric Perspective

- The Dialcctic Perspective

- The Evolutionary Perspective

- The Power Perspective

- The Fuller Argumentation Perspective

These are followed by some discussion of how to use these critique perspective to write your own article.

\section{Notes}

'The word "argument" is being used here in the sense of a one-line conclusion, proposition, conjecture or claim that needs to be justified by supporting evidence. It is not a quarrel, but wellreasoned; not pure logic but structured conversation.

${ }^{2}$ http://www. fas.harvard. edu/ wricntr/html/tools.htm.

${ }^{3}$ Examples provided by Colin East.

\section{References}

Ackoff R. (2002) www.judglink.org

Argyris C. and Schon D.A. (1996) Organisational Learning II, Boston, Mass: AddisonWesley.

Arygris C. (1996) Actionable Knowledge, Journal of Applied Behavioural Science, Vol 32(4), pp.390-406. 
Boulding K. (1961) General Systems Theory: The Skeleton of Science. Management Science, Vol 2 (3), pp.197-208.

Bronte-Stewart, M. (1999) Regarding Rich Pictures as Tools for Communication in Information Systems Development, Computing and Information Systems, Vol 6, pp. 83-102.

Checkland, P. (2000) "Soft Systems Methodology: A Thirty Year Retrospective", Systems Research and Behavioural Science, Vol 17 (1), pp. S11-S58.

Churchman, C.W. (1971) The Design of Inquiring Systems, New York: Wiley.

Churchman C.W. (1979) The Systems Approach and Its Enemies, New York: Basic Books.

Eemeren, van F.H., Grootendorst, R. and Kruiger, T. (1987) Handbook of Argumentation Theory, Dordrecht: Foris Publications.

Einstein A. (1995) Relativity: the Special and the General Theory, Crown Publications.

Haynes J. (2000) Perspectival Thinking, NZ: OneBook and Company.

Kuhn T.S. (1970) The Structure of Scientific Revolutions, 2nd Ed., Chicago: University of Chicago Press.

Linstone, H. (1984) Multiple Perspectives for Decision Making: Bridging the Gap Between Analysis and Action, New York, NY: Elsevier Science Publishing Co.

Linstone H. A. (1999) Decision Making for Technology Executives : Using Multiple Perspectives, Boston: Artech House Publishing.

Mason, R. O. (1969) “A Dialectical Approach to Strategic Planning”, Management Science, Vol.15, pp.B403-B414.

Mitroff I. and Linstone H. (1993) The Unbounded Mind, Oxford University Press.

Morgan G. (1986) Images of Organisations, Calif.: Sage Publications.

Nielsen R. P. (1996) Varieties of Dialectic Change Processes," Journal of Management Inquiry, 5, 4, September, 1996, pp. 276-292.

Perelman Ch. and Olbrechts-Tyteca L. (1969) The New Rhetoric: A Treatise on Argumentation, University of Notre Dame.

Popper K.R. (1963) Conjectures and Refutations, London: Routledge.

Sowell T. (1985) Marxism, London: Unwin.

Walton D. (1998) The New Dialectic, Toronto: Toronto University Press.

Weick K.E. (1983) 'Managerial Thought in the Context of Action', in The Executive Mind, eds. W. Bennis, R.O. Mason and I.I. Mitroff, Calif: Jossey Bass. 